\title{
Thermal convection instability of two miscible viscous fluids in a Hele-Shaw cell
}

\section{ECHCHADLI}

\section{University Hassan II of Casablanca, Faculty of Sciences Aïn-Chock, Laboratory of Mechanics, Casablanca, Morocco} mourad.echchadli@gmail.com

\begin{abstract}
:
The onset of convection in two superimposed miscible fluid layers is investigated in the configuration of a geometric Hele-Shaw cell using linear stability analysis. The two fluids have different densities. We neglect the surface tension and chemical diffusion at the interface which is assumed of small amplitude. We consider only the asymptotic case, where the Prandtl number's order is of the order of unity or larger than unity. We show, in the Hele-Shaw configuration, which can simulate convection in porous media, that the onset of convection can be either stationary or oscillatory depending on the Buoyancy number, $B$ (the ratio of the stabilizing chemical density anomaly to the destabilizing thermal density anomaly), which depends on the viscosity and layer height ratios. When the buoyancy number is lower than a critical value, $B_{c}$, oscillating convection occurs in the whole cell height, however beyond $B_{c}$, the stratified regime develops without deformation of the interface and convection occurs separately in each layer. In this paper, the transition from oscillatory regime to stratified regime is visualised by using the streamlines at the onset of convection
\end{abstract}

Keywords : Thermal convection; Two superimposed fluid layers, Miscible fluids; Hele-Shaw cell; Buoyancy number

\section{Introduction}

Several works have been devoted to the thermal instability in two chemically stratified fluid layer and these studies have shown that the onset of instability can be either steady or oscillatory [1]. The situation corresponding to the earth mantles where the interface remains flat and a stationary convection develops in two superimposed layers, was studied by several researchers [1]. Other theoretical and experimental works have shown that the addition of an intrinsic density difference, due to chemical differences between layers, can lead to an oscillatory convection which can also occur separately in the two superimposed fluid layers without deformation of the interface [2]. In the present study, we perform a linear stability analysis of a system, consisting of two miscible fluid layers, confined in a Hele-Shaw cell and submitted to a vertical temperature gradient. A first motivation to work in the geometric configuration of Hele-Shaw is to simulate flows in porous media. Indeed, by defining a permeability to the cell, being the distance between the vertical walls of the cell, several works have shown the existence of an analogy between convective flow in Hele-Shaw cell and that in a porous medium. A second motivation is that the Hele-Shaw cell allows us to be in the considerations assumed in [1], [3], where studies have considered a large Rayleigh number and infinite Prandtl number fluids. Indeed, in Hele-Shaw cell, a dimensionnal analysis and an asymptotic study lead to two formulations [4] : $\operatorname{Pr}=O(1)$ or $\operatorname{Pr}>>1$ and $\operatorname{Pr}=O\left(\epsilon^{2}\right)$, where $\epsilon$ is aspect ratio, which is smaller than unity. With the first formulation, we are exactly in the hypothesis, considered theoretically and experimentally by [1], [3] . We focus on the effects of viscosity and height ratios on the transition from oscillatory regime to stratified regime.

\section{MATHEMATICAL FORMULATION}

\subsection{Governing equations}

In the three-dimensional $x^{*}, y^{*}, z^{*}$ space, we consider two superimposed fluid layers, respectively of densities $\rho_{1 o}$ and $\rho_{2 o}$, kinematic viscosities $\nu_{1}$ and $\nu_{2}$ (dynamic viscosities $\mu_{1}$ and $\mu_{2}$ ), and height $d_{1}$ and $d_{2}$, confined in a horizontal Hele-Shaw cell of infinite extent in the $x$ direction. We denote by $d=d_{1}+d_{2}$ the height of the cell, $e$ the distance between the vertical planes and $\varepsilon=e / d<<1$ the aspect ratio of the cell. We assume that the two superimposed layers of fluids confined in the cell are bounded vertically by two thermally insulating walls located at $y^{*} \pm e / 2$ and horizontally by two perfect heat conducting plates located respectively at $z^{*}=-d_{1}, z^{*}=d_{2}$ and having constant temperatures $T_{1}$ and $T_{2}$.

Hereafter, we follow the dimensional analysis and scales used in the work of [4]. The linearized equation in the Hele-Shaw approximation and in normal mods [4] is written as :

$$
\begin{gathered}
s\left(D^{2}-k^{2}\right) \widetilde{T}_{1 o}=\left(D^{2}-k^{2}\right)^{2} \widetilde{T}_{1 o}-\frac{k^{2}}{12 \gamma} R a \widetilde{T}_{1 o} \\
s\left(D^{2}-k^{2}\right) \widetilde{T}_{2 o}=\left(D^{2}-k^{2}\right)^{2} \widetilde{T}_{2 o}-\frac{k^{2}}{12} R a \widetilde{T}_{2 o}
\end{gathered}
$$


Where $s=\sigma+i \omega$. The imaginary part of $s$, denoted $\omega$, is the phase velocity of the perturbation. The growth rate of the perturbation is $\sigma$, the real part of $s$. The term $k$ is the wavenumber, $R a=\frac{\beta_{i} g\left(T_{1}-T_{2}\right) e^{2} d}{\nu_{2} \kappa_{2}}$ is the Rayleigh number of the cell. Hereafter, we denote the viscosity ratio by $\gamma=\frac{\nu_{1}}{\nu_{2}}$, and $D^{2}=\frac{d^{2}}{d z^{2}}$. The system of equations, (1)-(2), is subject to the following boundary conditions :

(a) Outer boundary conditions

$$
\begin{array}{ll}
\widetilde{T}_{1 o}=\left(D^{2}-k^{2}\right) \widetilde{T}_{1 o}=0 & z=-a \\
\widetilde{T}_{2 o}=\left(D^{2}-k^{2}\right) \widetilde{T}_{2 o}=0 & z=1-a
\end{array}
$$

(b) Continuity of vertical velocities

$$
\left(D^{2}-k^{2}\right) \widetilde{T}_{1 o}=\left(D^{2}-k^{2}\right) \widetilde{T}_{2 o} \quad z=0
$$

(c) Continuity of normal stress

$$
\begin{gathered}
s^{2} D\left(\rho \gamma \widetilde{T}_{1 o}-\widetilde{T}_{2 o}\right)-s\left(D^{3}-k^{2} D\right)\left(\rho \gamma \widetilde{T}_{1 o}-\widetilde{T}_{2 o}\right) \\
=-B \operatorname{Ra} k^{2}\left(s-\left(D^{2}-k^{2}\right)\right) \widetilde{T}_{1 o} \quad z=0
\end{gathered}
$$

where $B$ is the buoyancy number, the ratio of the stabilizing chemical density anomaly to the destabilizing thermal density anomaly : $B=\frac{\rho_{10}-\rho_{2 o}}{\beta \rho_{2 o}\left(T_{1}-T_{2}\right)}$.

(d) Continuity of temperature and of heat flux yields

$$
\begin{aligned}
& \widetilde{T}_{1 o}-\widetilde{T}_{2 o}=0 \quad z=0 \\
& D \widetilde{T}_{1 o}-D \widetilde{T}_{2 o}=0 \quad z=0
\end{aligned}
$$

The partial differential system (1)-(2), with the associated boundary conditions (3)-(8), is solved numerically for arbitrary wave numbers using a spectral collocation scheme.

\section{RESULTS AND DISCUSSION}

In the system (1)-(8) appear the following parameters $\gamma$, $s=\sigma+i \omega, a, B, R a$ and $k$. Also, only results for $\gamma \geq 1$ will be presented, which means that the bottom layer will be the most viscous. We focus attention on the marginal stability curves, $\sigma=0$, separating the stable and unstable regions. The onset of convection is considered stationary if $\omega=0$ and oscillatory if $\omega \neq 0$.

\subsection{Effect of the buoyancy number}

In figure 1, for $\gamma=1$ and for $B=0$ (no density jump at the interface), the convection occurs throughout the whole layer. The Oscillatory regime is presented for $B=0.1 \prec B_{c}=0.2153$ (figure 1), here the vertical velocity is maximum at the interface, leading to convection over the whole depth. On the other hand, when the buoyancy number increases, the streamlines start to be concentrated in each fluid layer. When $B \succ B_{c}$ (figure 1), convection develops above and below the interface with a wavelength comparable to one layer depth, this regime has a zero vertical velocity at the interface, and the stability of this stratified regime is independent of B. The cells formed in each layer rotate in the same direction, we are in the presence of a thermal coupling.
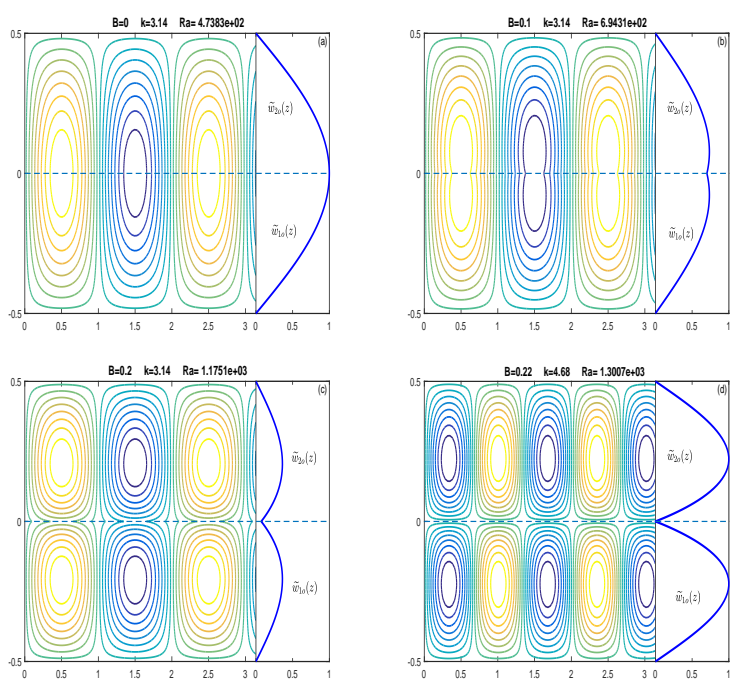

Figure 1 : Transition from the oscillatory regime to the stratified regime : streamlines and velocity profiles for $\gamma=1$ and for different values of the buoyancy number, $B$.

When $\gamma=6$, and for $B=0.0833$ (see figure 2), we are in the presence of an oscillatory regime where convection takes place over the whole height of the two-layer system. The streamlines are concentrated in the less viscous layer having a maximum velocity and the velocity profile in the lower layer is linear. By increasing the buoyancy number and for $B \succ B_{c}=0.136$, the regime is stratified and the velocity of the lower layer is near-zero. Only the upper layer is the place of convection.
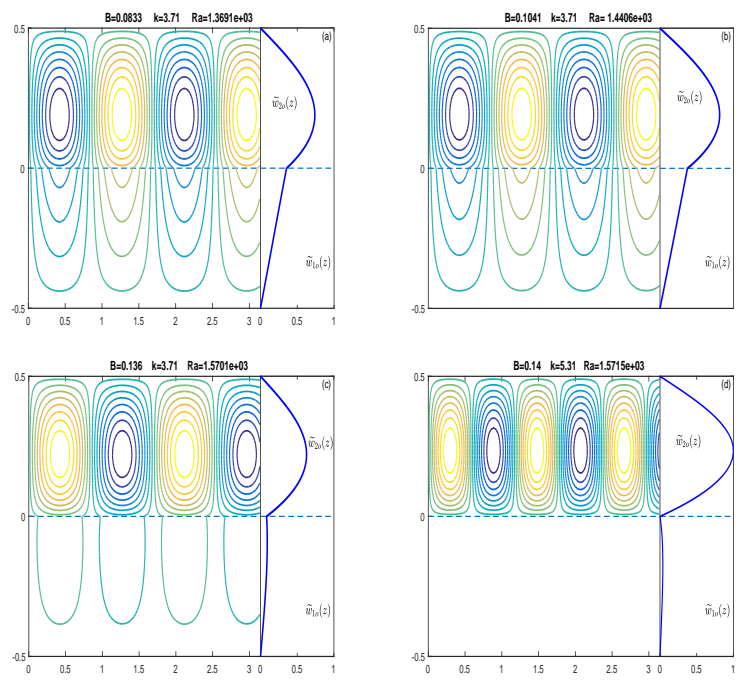

Figure 2 : Transition from the oscillatory regime to the stratified regime : streamlines and velocity profiles for $\gamma=6$. 


\subsection{Effects of the viscosity ratio and the depth ratio}

The effect of the viscosity ratio on the convection threshold is presented in figure 3 . We note that for $a=0.75$, the layer 1 is active because it is thicker than the second layer, this is illustrated in the figure $3(\gamma=1)$ where we have the lower layer having a maximum velocity and the convective cells are established in this layer. When the viscosity ratio is less than $10(\gamma<10)$ (see figure 3 ) for $\gamma=10$ ), it is easier for a viscous layer to cause the movement of the less viscous layer. Conversely, beyond $\gamma=10$, the layer 2 becomes active (see figure 3 for $\gamma=22$ and $\gamma=100$ ) and this transition is accompanied by a jump of the wavelength.
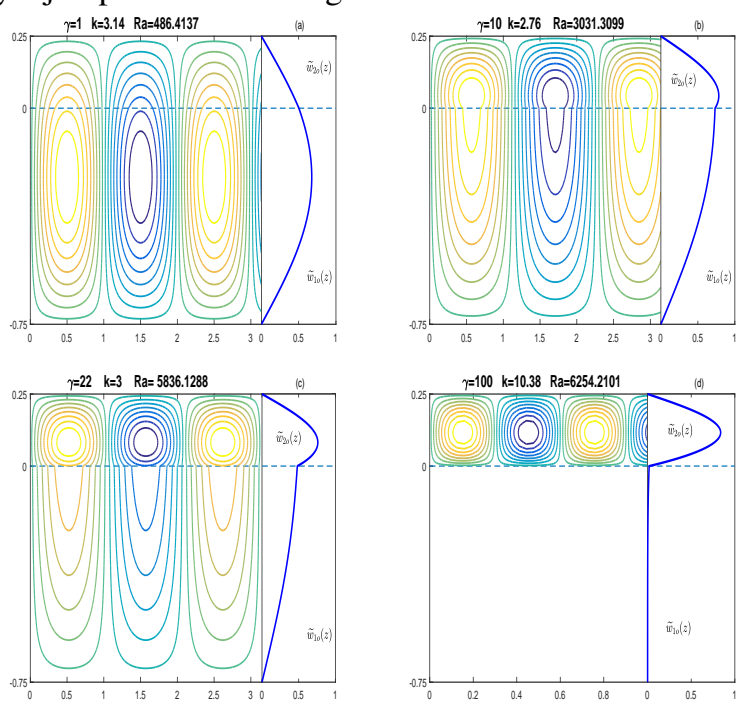

Figure 3: Streamlines and velocity profiles for $a=0.75, B=0.05$ and for different viscosity ratio.
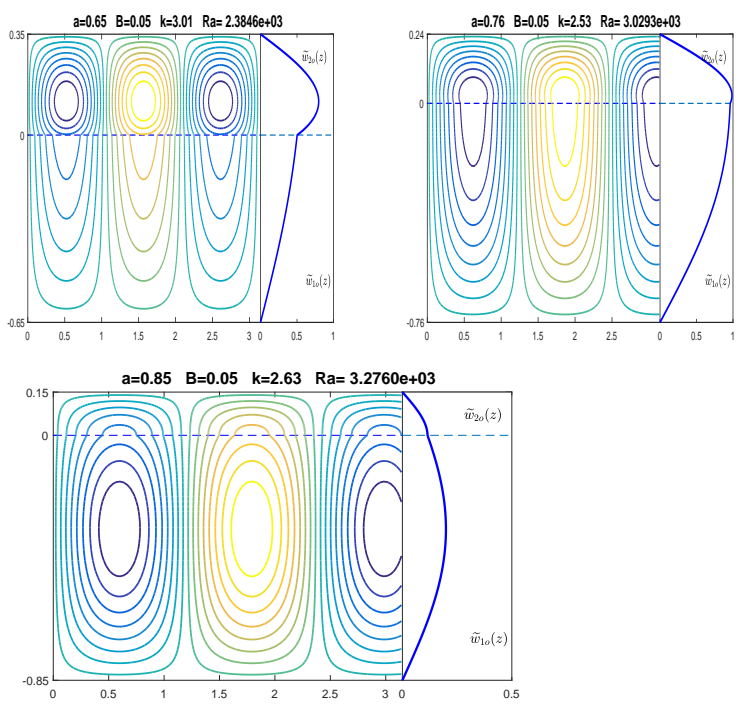

Figure 4: Streamlines and velocity profiles for $\gamma=10, B=0.05$ and for different depth ratio
The effect of the depth ratio on the convection threshold is presented in the figure 4 . When $\gamma=10$ and $B=0.05$, by varying $a$ from $a=0.65$ to $a=0.85$, the layer 2 having a maximum velocity, is active for $a=0.65$ and becomes passive when $a=0.85$. Similarly, the layer 1 that is passive for $a=0.65$ becomes active for $a=0.85$.

\section{CONCLUSION}

The influence of the contrast viscosity and that the buoyancy number on the linear stability of two-layer thermal convection in the presence of stable density stratification has been investigated. Depending on the buoyancy number, the ratio of the stabilizing chemical density anomaly to the destabilizing thermal density anomaly, two regimes are found :

(i) For $B<B_{c}(\gamma, a)$, we have an oscillatory regime where vertical motion exists at the interface.

(ii) For $B>B_{c}(\gamma, a)$, we have a steady two-layer regime where there is no vertical motion at the interface.

These results were confirmed, in particular, by visualization of the streamlines. The influence of viscosity combined with the ratio of the heights of the two layers was examined. For low viscosity ratios, and for $B \prec B_{c}$ and for $a=0.75$, convection occurs over the whole height of the fluid layer and the layer 1 is active while the layer 2 is passive. By increasing the viscosity ratio, the layer 2 becomes active and the layer 1 becomes passive. The illustration of these results was done by visualizing the streamlines by setting $a=0.75$ and varying the viscosity ratio.

\section{Références}

[1] F. M. Richter, et C. E Johnson, "Stability of a chemically layered mantle", J. Geophys. Res. 79, 1635 1639 (1974).

[2] S. Rasenat, F. H. Busse, et I. Rehberg, "A theoretical and experimental study of double-layer convection", J. Fluid Mech. 199 519-540 (1989).

[3] M. Le Bars, A. Davaille, "Stability of thermal convection in two superimposed miscible viscous fluids", J. Fluid Mech, 471,(2002) 339-363.

[4] S. Aniss , M. Souhar and M. Belhaq, "Asymptotic study of the convective parametric instability in hele-shaw cell", Phys. Fluids A 12, 262-268 (2000).

[5] C. Jaupart, P. Molnar and E. Cottrell, "Instability of a chemically dense layer heated from below and overlain by a deep, less viscous fluid", J. Fluid Mech., 572, 433-469 (2007). 